\title{
Devenir meilleure que le Dr Google
}

\section{Christina Aus der Au}

Prof. et Dre en théol., membre de la rédaction Ethique

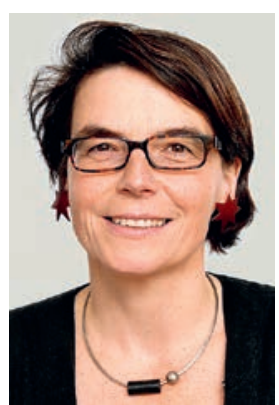

Une recherche avec le mot-clé «TDAH» dans Google donne plus de 9 millions de résultats, presque autant que les termes «dépression» et "adolescents», sans compter les illustrations, images, bandes dessinées, photos et statistiques. En tapant «enfants + troubles mentaux + symptômes», il y aura même plus de 26 millions en français et 160 millions en anglais.

Une pléthore d'informations destinées à des parents inquiets dont l'enfant vient d'être diagnostiqué hyperactif par la psychologue scolaire et qui, une fois rentrés chez eux, consultent le Dr Google pour comprendre ces termes pourtant employés avec la plus grande prudence. Peut-on leur en vouloir de s'informer ou de partager sur internet leurs expériences au contact de médecins, de psychologues ou de maladies?

Une de mes amies va devenir psychologue scolaire et son mémoire de Master traite justement de cette question. A quels résultats les parents ou les jeunes concernés sont-ils confrontés lorsqu'ils se lancent dans une recherche sur internet pour en savoir plus sur les troubles psychiques? Que découvrent-ils lorsqu'ils inscrivent certains mots-clés dans le moteur de recherche? Quelles informations objectives apparaissent, quelles théories du complot, quelles histoires réconfortantes ou angoissantes leur raconte internet?

Mon amie a donc épluché des centaines de pages, consulté des chats et analysé des images avec toujours la même question en tête: qu'ont vu et lu les parents avant de se rendre à leur prochaine consultation chez la psychologue scolaire? Elle doit tout à la fois être experte et néophyte. A quoi réagirait-elle, en tant que mère et que voudrait-elle savoir de plus? Et en tant que psychologue, comment comprend-elle ces pages? Qu'est-ce qui est absurde et inutile, quelles informations sont pertinentes, lesquelles sont dangereuses? Et comment expliquer la différence?

Prendre connaissance de toutes ces informations est une chose, se préparer aux questions et discussions en est une autre, en revanche il est certain que réfléchir à ce que le service de psychologie scolaire devrait faire pour que son site apparaisse dans les premiers résultats de recherche pour venir en aide aux parents et aux adolescents désemparés est une tâche à laquelle ses études ne l'ont pas préparée.

Je ne parle pas de rendre un site attrayant, une tâche pour laquelle il existe autant d'experts que pour la rédaction de contenus pertinents. La difficulté consiste à devoir agir sur plusieurs fronts. Etre expert, fournir des informations objectives et différenciées et se retrouver simultanément en concurrence avec des fournisseurs d'informations de tout bords qui parlent de manière plus claire, plus simple ou plus personnelle et semblent donc plus dignes de confiance.

Tom Nichols, un professeur américain pour les questions de sécurité nationale, a écrit un livre très pertinent à ce sujet intitulé «The Death of Expertise» [1]. Si, en tant que maman, je peux trouver des millions d'informations et de témoignages sur internet, pourquoi devrais-je accorder une plus grande importance à une seule page, alors qu'il y a des tas d'opinions différentes signées par des titulaires de doctorats? Si chaque doctrine est réfutée par de nombreux points de vue, pourquoi ne devrais-je pas croire celui qui correspond le mieux à ma vision du monde? Le sain scepticisme d'antan envers l'avis des demi-dieux en blanc s'est mué en foi aveugle en sa propre opinion, renforcée et démultipliée par l'écho et le filtre d'une communauté d'internautes qui la partagent.

Lorsque ses propres convictions sont contrecarrées par toutes celles et tous ceux qui nourrissent le web d'expériences subjectives, de ressentis sur le vif et de réactions émotionnelles, lorsque, au nom de l'autodétermination et de l'autonomie, chacun revendique équitablement le droit à sa propre opinion, je comprends aisément qu'un médecin ou une psychologue soit parfois pris de désespoir.

Mon amie consulte donc les pages internet, les forums, les sites d'informations et de groupes d'entraide. Elle s'immerge dans les théories du complot et le scepticisme scientifique tout en essayant de garder son esprit critique et de rester convaincante. Elle sait très bien qu'avoir raison ne suffit pas, il faut aussi savoir être entendue. Que tous les co-experts engagés et convaincus d'internet obtiennent plus de crédit, la motive à rester proche des gens que ce soit dans des discussions personnelles ou la jungle d'internet. 